\title{
Data-Driven Robust Control for a Closed-Loop
}

\section{Artificial Pancreas}

\section{N. Paoletti ${ }^{1}$, K.S. Liư ${ }^{2}$, H. Chen ${ }^{3}$, S.A. Smolka², S. Lin ${ }^{3}$}

1Department of Computer Science, Royal Holloway, University of London

2Department of Computer Science, Stony Brook University

${ }^{3}$ Department of Electrical and Computer Engineering, Stony Brook University

\section{Type 1 diabetes treatment}

- Type 1 diabetes (T1D) is an autoimmune diseases where the body cannot produce enough insulin to regulate blood glucose (BG)

- Current treatment consists of an insulin pump (continuous infusion) and a glucose monitor (CGM).

- But these do not communicate with each other, with the patient required to manually manage insulin dosing after each meal.

\section{Robust artificial pancreas}

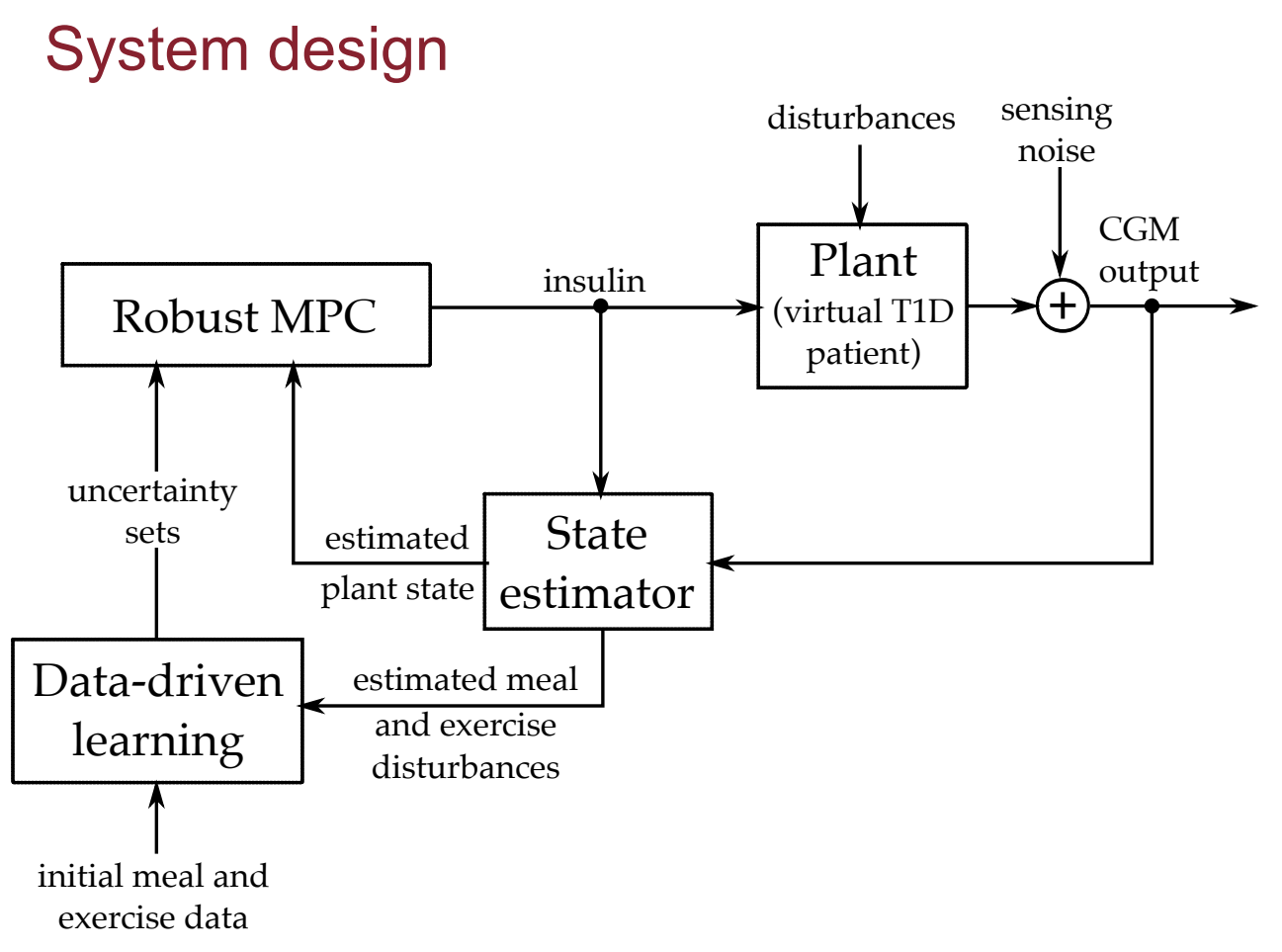

\section{Construction of uncertainty sets}
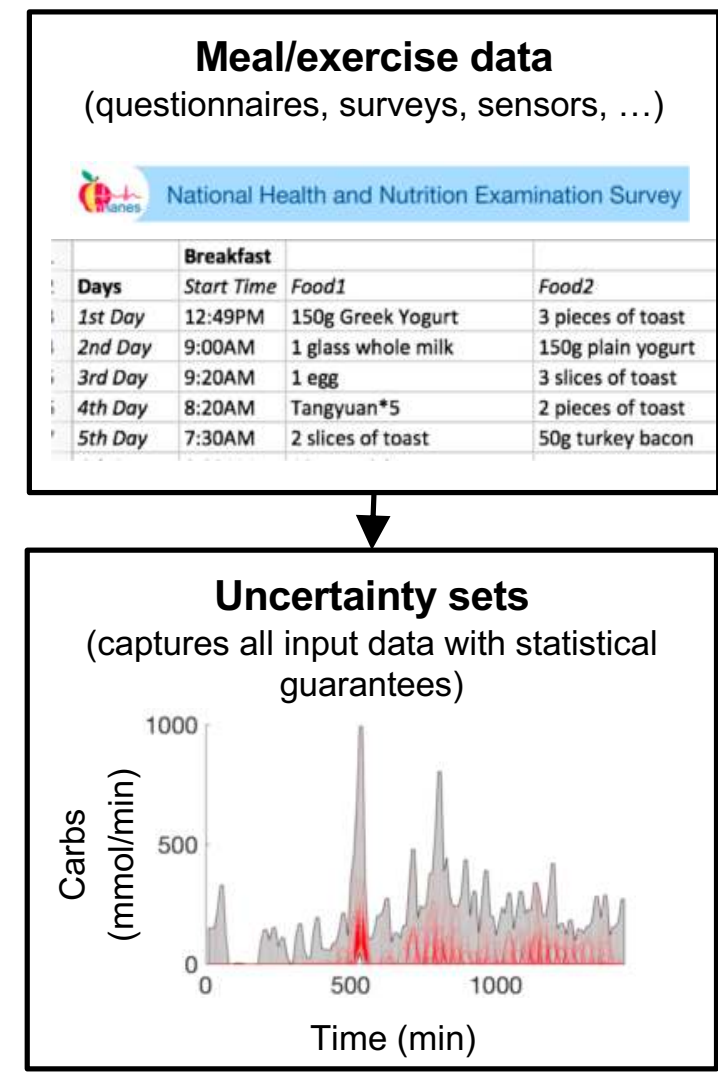

Bertsimas et al., Mathematical Programming (2013): 1-58

\section{Robust MPC}

Finds the insulin therapy at time $t, t+1, \ldots$ that minimizes the worst case performance w.r.t. unknown disturbances (bounded by the uncertainty sets)

$\min _{u^{t}, \ldots, u^{t+N_{c}-1}} \max _{\mathbf{d}^{t}, \ldots, \mathbf{d}^{t+N_{p}-1}} \sum_{i=1}^{N_{p}} d(\tilde{\mathbf{x}}(t+k))+\beta \cdot \sum_{k=0}^{N_{c}-1}\left(\Delta u^{t+k}\right)^{2}$

Performance: combination of distance from target trajectory and step-wise discrepancy of control strategy Moving-horizon state estimator

- It uses a model to minimize distance between predicted and actual measurements, and between predicted and estimated states over a moving window of length $\mathrm{N}$

- It also estimates the most-likely disturbances

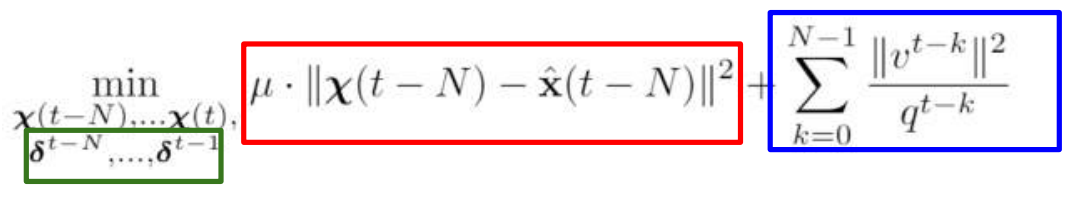

\section{Results}

One-meal experiments

We reproduce synthetic scenarios where

1) uncertainty sets are accurate w.r.t. random meals

2) uncertainty sets are accurate, but meals behave as outliers

3) random meals occur with constant delay

4) random meal followed by random exercise

Our robust controller keeps BG in range for $78 \%$ to $98 \%$ of the time

1) Accurate

2) Outliers

3) Delay
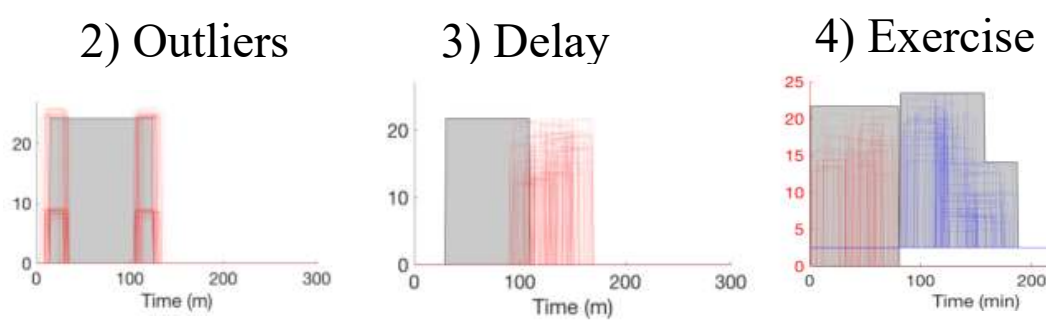

Carbs: Uncertainty sets (grey), random meals and oxygen due to exercise
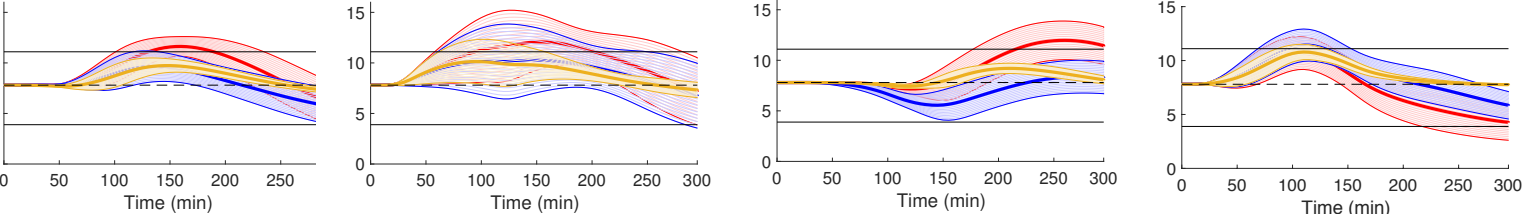

G: our robust controller compared to an ideal controller with exact knowledge of plant state and disturbances, and to a non-robust controller
Meal disturbances from CDC NHANES

- We constructed meal disturbance model from data of CDC NHANES database (8600+ records).

- We clustered the data into 10 main groups, for each deriving random meal disturbances and uncertainty sets.

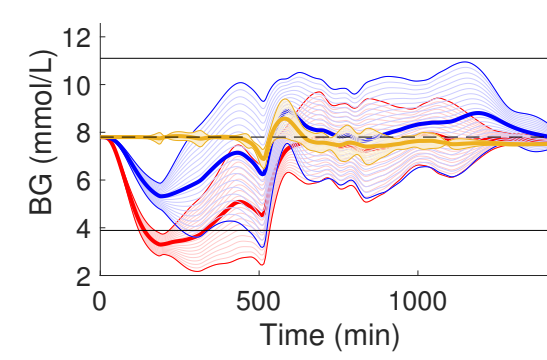

Random meals from NHANES data: robust controller keeps BG in range for $>95 \%$ of the time

\section{Random virtual patient} cohort + fixed meals from NHANES data: robust controller keeps BG in range for $>94 \%$ of the time
High-carbs intake

\begin{tabular}{llll}
\hline & Chance & $\begin{array}{l}\text { Carbs } \\
\text { (g) }\end{array}$ & $\begin{array}{l}\text { Time of day } \\
\text { (h) }\end{array}$ \\
\hline Breakfast & $100 \%$ & $40-60$ & $6: 00-10: 00$ \\
\hline Snack 1 & $50 \%$ & $5-25$ & $8: 00-11: 00$ \\
Lunch & $100 \%$ & $70-110$ & $11: 00-15: 00$ \\
Snack 2 & $50 \%$ & $5-25$ & $15: 00-18: 00$ \\
Dinner & $100 \%$ & $55-75$ & $18: 00-22: 00$ \\
Snack 3 & $50 \%$ & $5-15$ & $22: 00-00: 00$ \\
\hline
\end{tabular}

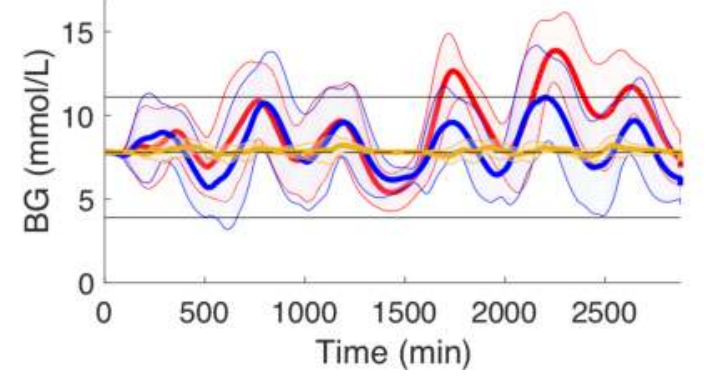

High-carbs intake: robust controller keeps BG in range for $81 \%$ of the time (against $70 \%$ of the non-robust controller) 